Article

\title{
Sequential MCR via Staudinger/Aza-Wittig versus Cycloaddition Reaction to Access Diversely Functionalized 1-Amino-1H-Imidazole-2(3H)-Thiones
}

\author{
Cecilia Ciccolini, Giacomo Mari, Gianfranco Favi*D, Fabio Mantellini, Lucia De Crescentini $\mathbb{D}$ \\ and Stefania Santeusanio *
}

Department of Biomolecular Sciences, Section of Chemistry and Pharmaceutical Technologies, University of Urbino “Carlo Bo", Via I Maggetti 24, 61029 Urbino (PU), Italy; c.ciccolini@campus.uniurb.it (C.C.); giacomo.mari@uniurb.it (G.M.); fabio.mantellini@uniurb.it (F.M.); lucia.decrescentini@uniurb.it (L.D.C.)

* Correspondence: gianfranco.favi@uniurb.it (G.F.); stefania.santeusanio@uniurb.it (S.S.)

Academic Editor: Wim Dehaen

Received: 25 September 2019; Accepted: 15 October 2019; Published: 21 October 2019

\begin{abstract}
A multicomponent reaction (MCR) strategy, alternative to the known cycloaddition reaction, towards variously substituted 1 -amino- $1 \mathrm{H}$-imidazole-2(3H)-thione derivatives has been successfully developed. The novel approach involves $\alpha$-halohydrazones whose azidation process followed by tandem Staudinger/aza-Wittig reaction with $\mathrm{CS}_{2}$ in a sequential MCR regioselectively leads to the target compounds avoiding the formation of the regioisomer iminothiazoline heterocycle. The approach can be applied to a range of differently substituted $\alpha$-halohydrazones bearing also electron-withdrawing groups confirming the wide scope and the substituent tolerance of the process for the synthesis of the target compounds. Interestingly, the concurrent presence of reactive functionalities in the scaffolds so obtained ensures post-modifications in view of $\mathrm{N}$-bridgeheaded heterobicyclic structures.
\end{abstract}

Keywords: multicomponent reaction; $\alpha$-halohydrazones; Staudinger reaction; aza-Wittig; $1 H$-imidazole2(3H)-thione; $2 H$-imidazo[2,1-b][1,3,4]thiadiazine

\section{Introduction}

Imidazoles belong to an important class of heterocyclic compounds that play a crucial role in various biochemical processes [1]. A lot of imidazole-based molecules have been shown bioactivities, [2] such as antifungal, antiinflammatory, antihystamine, antihelmintic, analgesic, antineoplastic, antihypertensive activity [3-7].

Among imidazole derivatives, imidazole-2-thiones have been associated to a special class of biologically relevant thiourea derivatives [8] endowed with antithyroid [9], antiproliferative [10], matrix metalloproteinases (MPP) inhibitory [11] properties and can be used as building blocks for the synthesis of $\mathrm{N}$-aminoimidazole with antiretroviral activity [12].

To date, the most widespread method used for the synthesis of $N$-substituted 1 -amino- $1 H$-imidazol2(3H)-thiones can be referred to the Schantl's protocol, which consists of reacting $\alpha$-haloketones with potassium thiocyanate and monosubstituted arylhydrazines in weak acidic medium (Scheme 1) [13-19]. This multistep reaction is considered to proceed via the formation of conjugated azoalkenes, derived from $\alpha$-thiocyanatohydrazones $\mathbf{D}$ (Scheme 2) and dipolarophile isothiocyanic acid intermediate that in turn undergo a [3+2] cycloaddition reaction providing substituted 1-arylamino-1 $\mathrm{H}$-imidazole-2(3H)-thione I scaffolds $[20,21]$. 

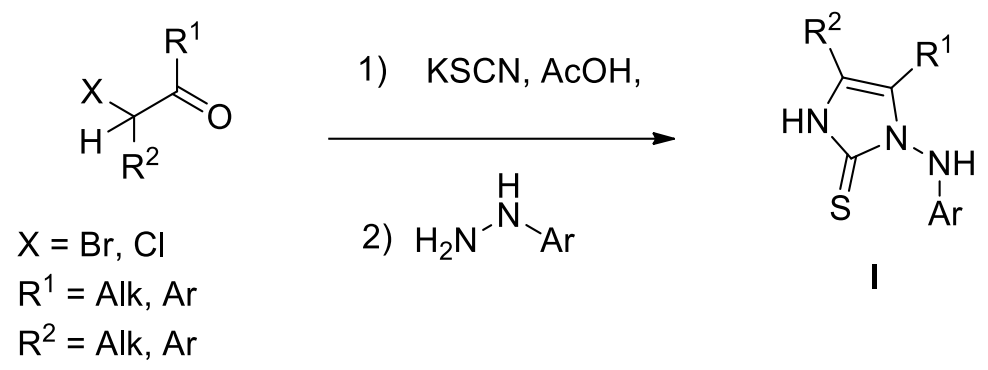

Scheme 1. Schantl's protocol for the synthesis of $N$-substituted 1-amino-1H-imidazole-2(3H)-thione derivatives $\mathbf{I}$.

Even if this method appears robust, it seems to suffer of some limitations in terms of insertion of electron-withdrawing groups placed on the $\alpha$-halohydrazone precursors of conjugated azoalkene intermediates. In this regard, for our research purposes, we tried to apply the Schantl's method reacting 2-chloro- $\mathrm{N}, \mathrm{N}$-dimethyl-3-oxobutanamide (A), potassiun thiocyanate (B) and tert-butyl hydrazinecarboxylate $(\mathrm{C})$ in acetic acid to obtain the corresponding $\mathrm{N}$-substituted 1 -amino- $1 \mathrm{H}$ imidazole-2(3H)-thione derivative I but without success. As shown in Scheme 2, instead of the cycloaddition, a 5-exo-dig cyclization reaction leading to 2-iminothiazole II took place. This evidence is in agreement with the result obtained by Lagoja and coworkers where a pathway involving the key $\alpha$-thiocyanatohydrazone intermediate $\mathbf{D}$ is invoked [12].<smiles>CC(=O)C(Cl)C(=O)N(C)C</smiles>

A

1) $\mathrm{KSCN}, \mathrm{AcOH}, \mathrm{rt}$
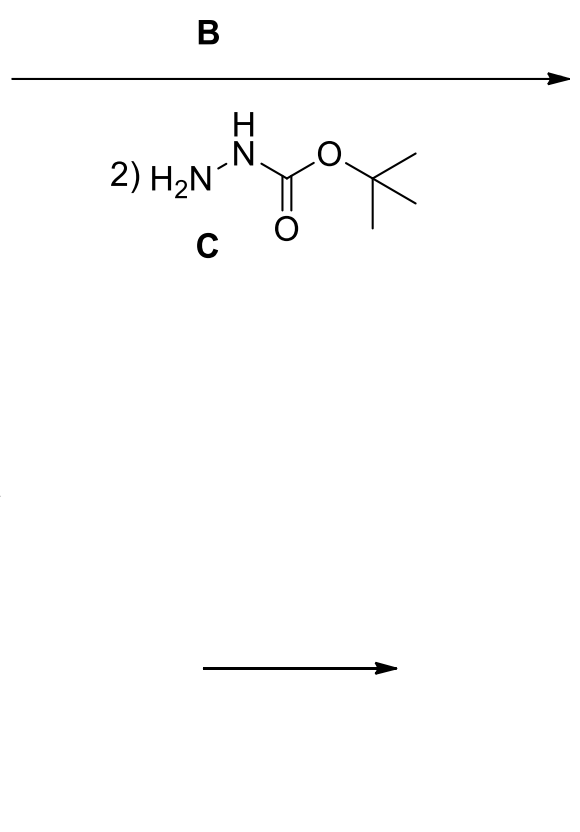<smiles>Cc1c(C(=O)N(C)C)sc(=N)n1NC(=O)OC(C)(C)C</smiles>

2-iminothiazoline II

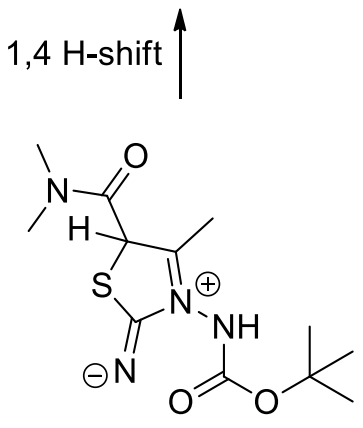

E

Scheme 2. Pathway for the formation of 2-iminothiazoline heterocycle II.

The structure of the iminothiazoline II was confirmed by comparison of the spectral data of the same compound obtained by means of a different procedure previously described by some of us that foresees the conjugated hydrothiocyanation of the pertinent conjugated azoalkene in acidic medium followed by intramolecular cyclization [22].

Inspired by our previous experience [23], and in order to perform a complete regioselective-oriented method for the desired 1-amino-1H-imidazole-2(3H)-thiones I, we have planned a different strategy that avoids the use of bidentate-nucleophilic reagents such as the potassium thiocyanate. In the construction of I, three strategic disconnections between the N1-C2, C2-N3 and N3-C4 were hypothesized (Scheme 3). 


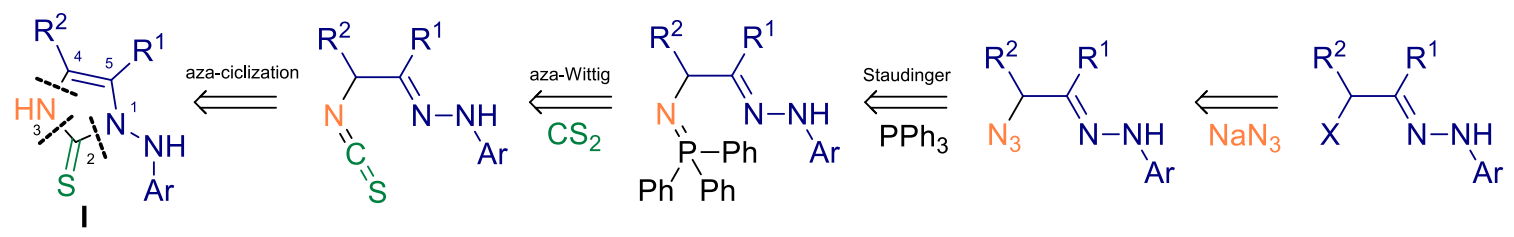

Scheme 3. Our hypothesized disconnection of 1-amino-1H-imidazole-2(3H)-thione I derivatives.

We reasoned that the azidation process of the pertinent $\alpha$-halohydrazone derivative followed by tandem Staudinger/aza-Wittig reaction with $\mathrm{CS}_{2}$ could have been a successful route [24,25].

\section{Results and Discussion}

To validate our hypothesis we began to explore the process step by step. Thus, $\alpha$-chlorohydrazone derivative 1a [26-30] (2.0 mmol) dissolved in THF $(9.0 \mathrm{~mL})$ subjected to $\alpha$-azidation using an ice-cooled aqueous solution of $\mathrm{NaN}_{3}$ [31] $(2.0 \mathrm{mmol} / 1.0 \mathrm{~mL})$ under magnetic stirring at room temperature. After the evaporation of the solvent and an appropriate extraction, the $\alpha$-azidohydrazone derivative 2a was obtained in 70\% yield. In the next step, the addition of a stoichiometric amount of $\mathrm{PPh}_{3}$ to $\mathbf{2 a}$ (1.0 mmol) dissolved in $\mathrm{CH}_{2} \mathrm{Cl}_{2}(5.0 \mathrm{~mL})$ furnished the iminophosphorane derivative 3a by precipitation from the reaction medium $(66 \%)$. Then, $3 a(0.65 \mathrm{mmol})$ was dissolved in $5.0 \mathrm{~mL}$ of THF/MeOH mixture (4:1) and treated with an excess of $\mathrm{CS}_{2}$ at reflux to afford, after column chromatography purification, the corresponding $N$-substituted 1-amino-2,3-dihydro- $1 H$-imidazole-2-thione derivative 5a (53\%) arising from intramolecular cyclization of the $\alpha$-isothiocyanate hydrazone intermediate $4 \mathbf{a}$ (Scheme 4 ).<smiles>C/C(=N\NC(=O)OC(C)(C)C)C(=O)N(C)C(=O)C(N)/C(C)=N/NC(=O)OC(C)(C)C(=O)N(C)C</smiles>

$1 a$<smiles>Cc1c(C(=O)N(C)C)[nH]c(=S)n1NC(=O)OC(C)(C)C</smiles>

$5 a$
$2 \mathbf{a}$

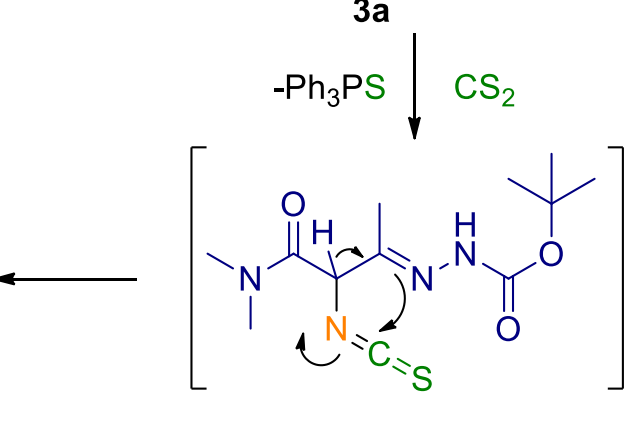

$4 a$

Scheme 4. Step-by-step synthetic pathway for $N$-substituted 1-amino-2,3-dihydro- $1 H$-imidazole-2thione derivative $\mathbf{5 a}$.

Motivated by this result, we aimed to develop a one-pot sequential multicomponent reaction (MCR) [32-35] as alternative method for regioselective synthesis of a new series of imidazole-2thione-containing structures as suitable precursors for drug-like compounds [36].

Hence, our new approach to $N$-substituted 1-amino- $1 H$-imidazole-2(3H)-thiones 5a-k (53\%-85\%) is depicted in Scheme 5. The whole process that permits the formation of the desired heterocycle can be easily checked by the complete disappearance of the pertinent $\alpha$-azidohydrazone derivative and by the observation of $\mathrm{Ph}_{3} \mathrm{P}=\mathrm{S}$ as byproduct (thin-layer chromatography (TLC) check, see Experimental Section). It is to be noted that for $\mathbf{5 a}$, the efficiency of the reaction benefits by this latter protocol increasing the overall yield from 25\% (obtained employing the step-by-step procedure) to $79 \%$ (Table 1). Moreover, the implemented strategy broadens the substitution patterns at the amino-N1 and at C4 of the 
heterocycle skeleton with electron-withdrawing groups (5a-e) and tolerates the aromatic (amino-N1) and aliphatic (C4) groups, as for $5 \mathbf{j}[15,17,18]$ (Table 1).<smiles>[R]NN=C([R])C([R])[X]</smiles>

1a-k
1) $\mathrm{NaN}_{3} ; \mathrm{H}_{2} \mathrm{O} / \mathrm{THF}(1 / 9 \mathrm{v} / \mathrm{v})$

2) $\mathrm{Na}_{2} \mathrm{SO}_{4} ; \mathrm{PPh}_{3} ; \mathrm{rt}$

3) $\mathrm{CS}_{2} ; \Delta$<smiles>[R16][R]Nn1c([R])c([R])[nH]c1=S</smiles>

Scheme 5. New multicomponent reaction (MCR) method for $\mathrm{N}$-substituted 1-amino-2,3-dihydro-1Himidazole-2-thione derivatives $\mathbf{5 a - k}$.

Table 1. Substrate scope of the MCR synthetic pathway for $\mathrm{N}$-substituted 1-amino-2,3-dihydro- $1 \mathrm{H}$ imidazole-2-thione derivatives $\mathbf{5 a - k}$.

\begin{tabular}{|c|c|c|c|c|c|c|c|}
\hline \multirow[t]{2}{*}{ Entry } & \multicolumn{5}{|c|}{$\alpha$-Halohydrazone 1} & & $\begin{array}{l}\text { One-Pot MCR } \\
\text { Yield }(\%) a, b\end{array}$ \\
\hline & & $\mathrm{R}^{1}$ & $\mathrm{R}^{2}$ & $\mathrm{R}^{3}$ & $x$ & & \\
\hline 1 & $1 \mathbf{a}$ & $\mathrm{CO}_{2} \mathrm{Bu}^{t}$ & $\mathrm{Me}$ & $\mathrm{CON}(\mathrm{Me})_{2}$ & $\mathrm{Cl}$ & $5 a$ & $25^{\mathrm{a}} ; 79^{\mathrm{b}}$ \\
\hline 2 & $1 b$ & CONHPh & $\mathrm{Me}$ & $\mathrm{CON}(\mathrm{Me})_{2}$ & $\mathrm{Cl}$ & $5 b$ & $53^{b}$ \\
\hline 3 & 1c & $\mathrm{CO}_{2} \mathrm{Bu}^{t}$ & $\mathrm{Me}$ & $\mathrm{CON}(\mathrm{Et})_{2}$ & $\mathrm{Br}$ & $5 c$ & $72^{b}$ \\
\hline 4 & 1d & $\mathrm{CO}_{2} \mathrm{Bu}^{t}$ & $\mathrm{Me}$ & $\mathrm{H}$ & $\mathrm{Cl}$ & $5 d$ & $69^{b}$ \\
\hline 5 & 1e & $\mathrm{CO}_{2} \mathrm{Bu}^{t}$ & $\mathrm{Me}$ & $\mathrm{CONH}_{2}$ & $\mathrm{Br}$ & $5 e$ & $58^{b}$ \\
\hline 6 & 1f & $\mathrm{CO}_{2} \mathrm{Bu}^{t}$ & $\mathrm{Me}$ & CONHPh & $\mathrm{Br}$ & $5 f$ & $67^{b}$ \\
\hline 7 & $1 \mathrm{~g}$ & CONHPh & $\mathrm{Me}$ & $\mathrm{H}$ & $\mathrm{Cl}$ & $5 g$ & $82^{b}$ \\
\hline 8 & 1h & $\mathrm{COPh}$ & $\mathrm{Me}$ & $\mathrm{H}$ & $\mathrm{Cl}$ & $5 \mathrm{~h}$ & $59^{b}$ \\
\hline 9 & $\mathbf{1 i}$ & CONHPh & $\mathrm{Me}$ & $\mathrm{Me}$ & $\mathrm{Cl}$ & $5 \mathbf{i}$ & $85^{b}$ \\
\hline 10 & $\mathbf{1 j}$ & $4-\mathrm{NO}_{2}-\mathrm{Ph}$ & $\mathrm{Me}$ & $\mathrm{Me}$ & $\mathrm{Cl}$ & $5 \mathbf{j}$ & $84^{b}$ \\
\hline 11 & $1 \mathrm{k}$ & $\mathrm{CO}_{2} \mathrm{Bu}^{t}$ & $\mathrm{Ph}$ & $\mathrm{H}$ & $\mathrm{Br}$ & $5 k$ & $66^{\mathrm{b}}$ \\
\hline
\end{tabular}

${ }^{a}$ Overall yield of isolated product $\mathbf{5 a}$ from the step-by-step reaction based on $\mathbf{1 a} ;{ }^{\mathbf{b}}$ Overall yield of isolated products 5a-k from one-pot MCR based on $\mathbf{1 a}-\mathbf{k}$.

These results not only lie in the wide scenario of the heterocyclic scaffolds obtainable through tandem Staudinger/aza-Wittig sequence [24,25,37-41], but the concurrent presence of reactive functionalities in the target compounds $\mathbf{5 a - k}$ ensures post-modifications in view of heterobicyclic structures. In fact, the tautomerism thionoamide/thioloimide permits the introduction of a further element of diversity at the sulfur atom producing imidazole derivatives suitable to be combined with the useful 1-amino-Boc protected group [42] directly installed by this approach, as for $\mathbf{5 a}, \mathbf{5} \mathbf{c}-\mathbf{f}, \mathbf{5 k}$. Thus, as an example, $\mathbf{5} \mathbf{c}, \mathbf{d}, \mathbf{f}(1.0 \mathrm{mmol})$ solved in acetone $(10.0 \mathrm{~mL})$, were reacted with 2-bromo-1-phenylethanone (6a) $(1.0 \mathrm{mmol})$, 1-chloropropan-2-one) $(6 \mathbf{b})(1.0 \mathrm{mmol})$, and ethyl 2-bromoacetate $(6 \mathbf{c})(1.0 \mathrm{mmol})$, respectively, in the presence of $\mathrm{K}_{2} \mathrm{CO}_{3}(1.0 \mathrm{mmol})$. After the removal of solvent followed by extraction, the corresponding $\alpha$-(imidazol-2-ylthio) carbonyl compounds $7 \mathbf{a}-\mathbf{c}$ were obtained as solid after column chromatography purification (84\%-93\%) (Scheme 6). The subsequent cleavage of the Boc-protecting group under homogeneous [43] or heterogeneous acidic conditions [44] was able to produce free amino function available to interact with the carbonyl appendage in 2-position of the ring, affording new $2 \mathrm{H}$-imidazo $[2,1-b][1,3,4]$ thiadiazine derivatives $\mathbf{8 a}, \mathbf{b}$ by condensation or $2 \mathrm{H}$-imidazo[2,1-b][1,3,4]thiadiazinone derivative $8 \mathrm{c}$ by acylic nucleophilic substitution process (Scheme 6, Table 2). 


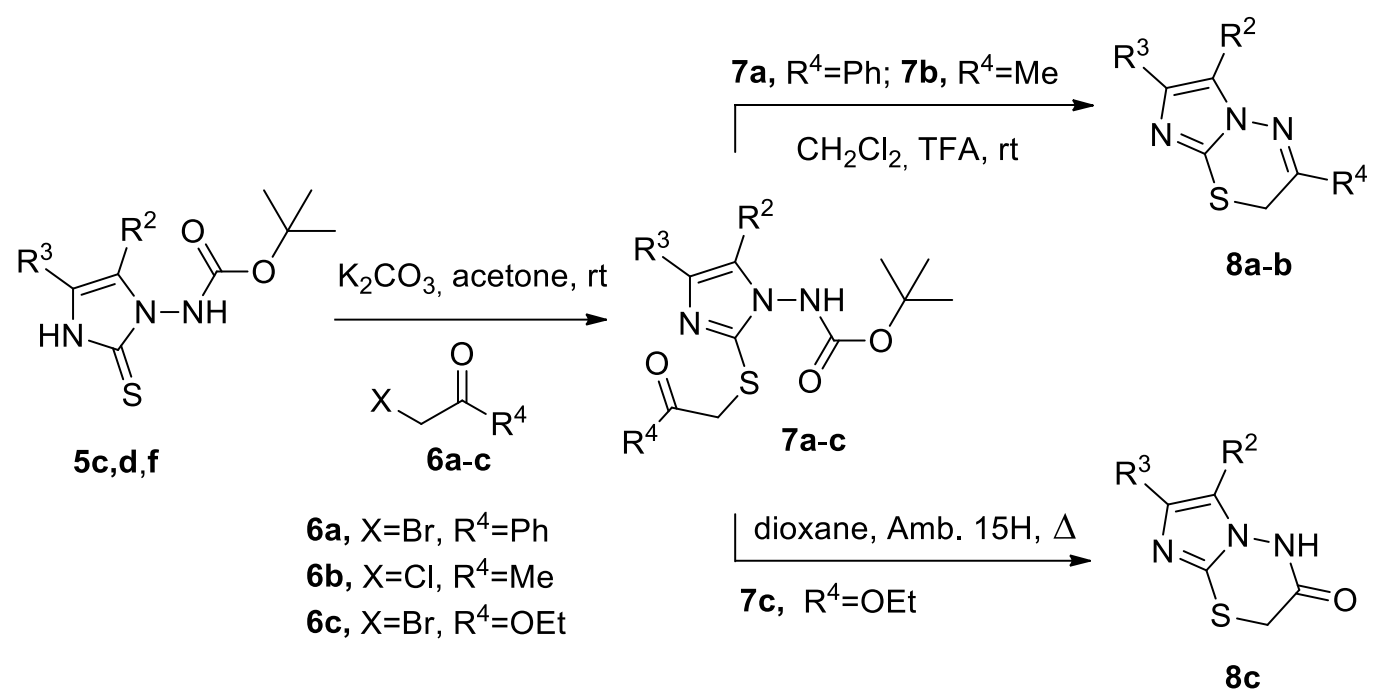

Scheme 6. Synthetic approach to $2 H$-imidazo[2,1- $b][1,3,4]$ thiadiazine derivatives.

Table 2. Substrate scope of the reaction between 1-amino-2,3-dihydro- $1 H$-imidazole-2-thione derivatives 5 with $\alpha$-haloketones $\mathbf{6 a}, \mathbf{b}$ or $\alpha$-haloester $\mathbf{6 c}$.

\begin{tabular}{|c|c|c|c|c|c|c|c|c|c|}
\hline & \multicolumn{2}{|c|}{5} & & \multicolumn{2}{|c|}{6} & \multirow[t]{2}{*}{7} & \multirow[t]{2}{*}{ Yield (\%) ${ }^{a}$} & \multirow[t]{2}{*}{8} & \multirow[t]{2}{*}{ Yield (\%) ${ }^{b}$} \\
\hline & $\mathrm{R}^{2}$ & $\mathrm{R}^{3}$ & & $x$ & $\mathrm{R}^{4}$ & & & & \\
\hline $5 c$ & $\mathrm{Me}$ & $\mathrm{CON}(\mathrm{Et})_{2}$ & $6 a$ & $\mathrm{Br}$ & $\mathrm{Ph}$ & $7 a$ & 84 & $8 a$ & 82 \\
\hline $5 d$ & $\mathrm{Me}$ & $\mathrm{H}$ & $6 b$ & $\mathrm{Cl}$ & $\mathrm{Me}$ & $7 b$ & 93 & $8 b$ & 65 \\
\hline $5 f$ & $\mathrm{Me}$ & CONHPh & $6 c$ & $\mathrm{Br}$ & OEt & $7 c$ & 92 & $8 c$ & 74 \\
\hline
\end{tabular}

${ }^{a}$ Yield of isolated product $7 \mathbf{a}-\mathbf{c}$ based on $\mathbf{6 a}-\mathbf{c}$; ${ }^{b}$ Yield of isolated product $8 \mathbf{a}-\mathbf{c}$ based on $7 \mathbf{a}-\mathbf{c}$.

It is worthwhile to note that the proposed synthetic pathway can offer an alternative method for obtaining $2 H$-imidazo[2,1- $b][1,3,4]$ thiadiazine derivatives 8 with respect to the ring transformation of $\alpha$-(oxazol-2-ylthio) ketones 9 on treatment with hydrazine hydrate 10 [45], together with the possibility of wide diversification of the substituents at the different positions of the $N$-bridgeheaded heterobicyclic structures. As depicted in Scheme 7, a different disconnection for the assembly of the $2 H$-imidazo[2,1- $b][1,3,4]$ thiadiazine scaffold can be envisaged.<smiles>[R]c1[nH]c(=S)n(NC(=O)OC(C)(C)C)c1[R]</smiles>

5

$\mathrm{R}^{2}=\mathrm{Me}$

$\mathrm{R}^{3}=\mathrm{H}, \mathrm{CON}(\mathrm{Et})_{2}, \mathrm{CONHPh}$<smiles>[R]c1nc(SCC(=O)[Z10]N)oc1[R]</smiles>

10
Ref 25

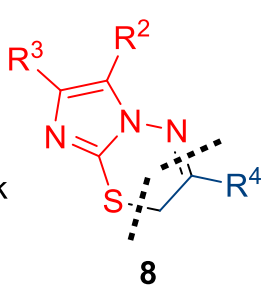

$$
8
$$

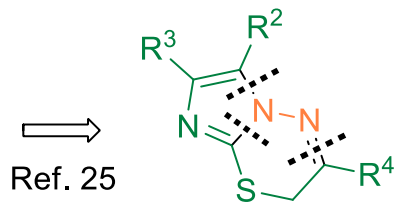

8

$$
\mathrm{R}^{2}=\mathrm{R}^{3}=\mathrm{Ar}
$$

Scheme 7. Different synthetic approaches to $2 H$-imidazo[2,1-b][1,3,4]thiadiazine derivatives. 


\section{Experimental Section}

\subsection{General}

All the commercially available reagents and solvents were used without further purification. $\alpha$-Halohydrazones 1a-k were synthesized by known procedures [26-30]. Chromatographic purification of compounds was carried out on silica gel (60-200 $\mu \mathrm{m})$. Thin-layer chromatography (TLC) analysis was performed on pre-loaded $(0.25 \mathrm{~mm})$ glass supported silica gel plates (Silica gel 60, F254, Merck; Darmstadt, Germany); compounds were visualized by exposure to UV light. Melting points (Mp) were determined in open capillary tubes and are uncorrected.

All ${ }^{1} \mathrm{H}$ NMR and ${ }^{13} \mathrm{C} \mathrm{NMR} \mathrm{spectra} \mathrm{were} \mathrm{recorded} \mathrm{at} 400$ and $100 \mathrm{MHz}$, respectively at $25{ }^{\circ} \mathrm{C}$ on a Bruker Ultrashield 400 spectrometer (Bruker, Billerica, MA, USA). Proton and carbon spectra were referenced internally to residual solvent signals as follows: $\delta=2.50$ ppm for proton (middle peak) and $\delta=39.50$ ppm for carbon (middle peak) in DMSO- $d_{6}$ and $\delta=7.27$ ppm for proton and $\delta=77.00 \mathrm{ppm}$ for carbon (middle peak) in $\mathrm{CDCl}_{3}$. The following abbreviations are used to describe peak patterns where appropriate: $\mathrm{s}=$ singlet, $\mathrm{d}=$ doublet, $\mathrm{t}=$ triplet $\mathrm{q}=$ quartet, $\mathrm{m}=$ multiplet and $\mathrm{br}=$ broad signal. All coupling constants $(J)$ are given in $\mathrm{Hz}$. Copies of ${ }^{1} \mathrm{H}-\mathrm{NMR}$ and ${ }^{13} \mathrm{C}-\mathrm{NMR}$ spectra of compounds II, 2a, 3a, 5a-k, 7a-c, and $\mathbf{8 a - c}$ are in Supplementary Materials. FT-IR spectra were measured as Nujol mulls using a Nicolet Impact 400 (Thermo Scientific, Madison, WI, USA). Mass spectra were obtained by ESI-MS analyses performed on Thermo Scientific LCQ Fleet Ion Trap LC/MS and Xcalibur data System. High-resolution mass spectra (HRMS) were determined with ESI resource on a Waters Micromass QTOF instrument (Waters, Milford, MA, USA). Elemental analyses were within \pm 0.4 of the theoretical values $(\mathrm{C}, \mathrm{H}, \mathrm{N})$.

\subsection{Step-By-Step Synthetic Method for $5 a$}

\subsubsection{Synthesis of tert-butyl 2-(3-azido-4-(dimethylamino)-4-oxobutan-2-ylidene)} hydrazinecarboxylate (2a)

To the $\alpha$-halohydrazone $1 \mathrm{a}(555.5 \mathrm{mg}, 2.0 \mathrm{mmol})$ solved in THF $(9.0 \mathrm{~mL})$, an ice-cooled aqueous solution $\left(1.0 \mathrm{~mL}, \mathrm{~T}=4^{\circ} \mathrm{C}\right)$ of $\mathrm{NaN}_{3}(2.0 \mathrm{mmol}, 130.02 \mathrm{mg})$ was added. The reaction mixture was stirred at room temperature until the disappearance of the starting 1a (TLC check). THF was removed under reduced pressure and the residue was diluted with water and extracted with $\mathrm{CH}_{2} \mathrm{Cl}_{2}(3 \times 15.0 \mathrm{~mL})$. The combined organic layers were dried over anhydrous $\mathrm{NaSO}_{4}$ and concentrated under reduced pressure. The crude reaction was purified by crystallization from $\mathrm{Et}_{2} \mathrm{O}$ affording the $\alpha$-azido derivative 2a. Yield 70.0\% (398.0 mg) as a white solid; Mp 120-124 $\left.{ }^{\circ} \mathrm{C}(\mathrm{dec}) ;{ }^{1} \mathrm{H}-\mathrm{NMR}, 400 \mathrm{MHz}, \mathrm{DMSO}-d_{6}\right) \delta 1.44$ $\left(\mathrm{s}, 9 \mathrm{H}, \mathrm{OBu}^{t}\right), 1.84\left(\mathrm{~s}, 3 \mathrm{H}, \mathrm{CH}_{3}\right), 2.86\left(\mathrm{~s}, 3 \mathrm{H}, \mathrm{NCH}_{3}\right), 2.92\left(\mathrm{~s}, 3 \mathrm{H}, \mathrm{NCH}_{3}\right), 4.99(\mathrm{~s}, 1 \mathrm{H}, \mathrm{CH}), 9.82(\mathrm{br} \mathrm{s}, 1 \mathrm{H}$, $\mathrm{NH}, \mathrm{D}_{2} \mathrm{O}$ exch.); ${ }^{13} \mathrm{C}-\mathrm{NMR}\left(100 \mathrm{MHz}, \mathrm{DMSO}-d_{6}\right) \delta 13.8,28.0,35.5,36.6,64.6,79.5,146.2,152.9,166.5 ; \mathrm{IR}$ (Nujol, v, cm ${ }^{-1}$ ): 3239, 3150, 2982, 2172, 2098, 1706, 1686, 1664; MS m/z (ESI): 285.07 (M + H) ${ }^{+}$; anal. calcd. for $\mathrm{C}_{11} \mathrm{H}_{20} \mathrm{~N}_{6} \mathrm{O}_{3}$ (284.31): 46.47; H, 7.09; N, 29.56; found: C, 46.36; H, 7.15; N, 29.65.

3.2.2. Synthesis of tert-butyl 2-(4-(dimethylamino)-4-oxo-3-((triphenylphosphoranylidene)amino) butan-2-ylidene)hydrazinecarboxylate (3a)

$1.0 \mathrm{Mmol}$ of $2 \mathbf{a}(284.31 \mathrm{mg})$ was solved in $\mathrm{CH}_{2} \mathrm{Cl}_{2}(5.0 \mathrm{~mL})$. The reaction flask was then immersed in an ice bath $\left(\mathrm{T}=0{ }^{\circ} \mathrm{C}\right)$, and a cooled solution of $\mathrm{PPh}_{3}(262.3 \mathrm{mg}, 1.0 \mathrm{mmol})$ in $\mathrm{CH}_{2} \mathrm{Cl}_{2}(1.0 \mathrm{~mL})$ was added dropwise. The reaction was brought back to room temperature and stirred until the disappearance of organic azide 2a (monitored by TLC). The formation of phosphazene 3a was accompanied by the development of $\mathrm{N}_{2}$. After partial removal of the solvent under reduced pressure, 3a was isolated by precipitation from a solution of $\mathrm{CH}_{2} \mathrm{Cl}_{2} /$ EtOAc as white powder; yield $66 \%(342.3 \mathrm{mg}) ; \mathrm{Mp} 127-131{ }^{\circ} \mathrm{C}$ (dec.); ${ }^{1} \mathrm{H}-\mathrm{NMR}\left(400 \mathrm{MHz}, \mathrm{DMSO}-d_{6}\right) \delta 1.42\left(\mathrm{~s}, 9 \mathrm{H}, \mathrm{OBu}^{t}\right), 1.81\left(\mathrm{~s}, 3 \mathrm{H}, \mathrm{CH}_{3}\right), 2.59\left(\mathrm{~s}, 3 \mathrm{H}, \mathrm{NCH}_{3}\right)$, $2.73\left(\mathrm{~s}, 3 \mathrm{H}, \mathrm{NCH}_{3}\right), 4.62\left(\mathrm{t}, \mathrm{J}_{\mathrm{H}-\mathrm{P}}=9.2 \mathrm{~Hz}, 1 \mathrm{H}, \mathrm{CH}\right), 7.57-7.90(\mathrm{~m}, 15 \mathrm{H}, \mathrm{Ar}), 9.64\left(\mathrm{~s}, 1 \mathrm{H}, \mathrm{NH}, \mathrm{D}_{2} \mathrm{O}\right.$ exch.) ppm; ${ }^{13} \mathrm{C}-\mathrm{NMR}\left(100 \mathrm{MHz}, \mathrm{DMSO}-d_{6}\right) \delta 13.0,28.0,35.6,36.1,59.3,79.5,120.9\left({ }^{1} J_{\mathrm{C}-\mathrm{P}}=102.0 \mathrm{~Hz}\right)$, 
$129.7\left({ }^{2} J_{\mathrm{C}-\mathrm{P}}=14.0 \mathrm{~Hz}\right), 133.7\left({ }^{3} J_{\mathrm{C}-\mathrm{P}}=11.0 \mathrm{~Hz}\right), 133.8\left({ }^{3} \mathrm{~J}_{\mathrm{CP}}=12.0 \mathrm{~Hz}\right), 134.9\left({ }^{4} J_{\mathrm{C}-\mathrm{P}}=2.0 \mathrm{~Hz}\right), 150.7$, 167.0 ppm; IR (Nujol, $v, \mathrm{~cm}^{-1}$ ): 3543, 3377, 3211, 1722, 1664; MS m/z (ESI): $519.31(\mathrm{M}+\mathrm{H})^{+}$; anal. calcd. for $\mathrm{C}_{29} \mathrm{H}_{35} \mathrm{~N}_{4} \mathrm{O}_{3} \mathrm{P}$ (518.59): $\mathrm{C}, 67.17 ; \mathrm{H}, 6.80 ; \mathrm{N}, 10.80$; found: $\mathrm{C}, 67.31 ; \mathrm{H}, 6.86 ; \mathrm{N}, 10.72$.

3.2.3. Synthesis of tert-butyl (4-(dimethylcarbamoyl)-5-methyl-2-thioxo-2,3-dihydro-1H-imidazol-1yl)carbamate (5a)

$0.65 \mathrm{Mmol}$ of $3 \mathbf{a}(337.0 \mathrm{mg}$,) was solved in a mixture of THF:MeOH $(4: 1,5.0 \mathrm{~mL})$ heating. Then, $0.5 \mathrm{~mL}$ of $\mathrm{CS}_{2}$ was added and the reaction was refluxed. The end of the reaction was defined $(4.0 \mathrm{~h})$ by the disappearance of $3 \mathbf{a}$ together with the formation of $\mathrm{Ph}_{3} \mathrm{P}=\mathrm{S}$ as byproduct (monitored by TLC). After removal of the reaction solvents under reduced pressure, a first crop of $5 \mathbf{a}$ was obtained as white powder from a solution of THF/light petroleum ether. A further amount was be gained by column chromatography eluting with $\mathrm{CH}_{2} \mathrm{Cl}_{2} / \mathrm{EtOAc}$ mixtures. White powder from THF/light petroleum ether; yield 53\% (103.4 mg); Mp 172-173 ${ }^{\circ} \mathrm{C}$ (dec.); ${ }^{1} \mathrm{H}-\mathrm{NMR}\left(400 \mathrm{MHz}, \mathrm{DMSO}-d_{6},\right) \delta 1.32$ and $1.45(2 \mathrm{~s}, 9 \mathrm{H}$, $\left.\mathrm{OBu}^{t}\right), 1.99\left(\mathrm{~s}, 3 \mathrm{H}, \mathrm{CH}_{3}\right), 2.94$ [s, 6H, N( $\left.\left.\mathrm{CH}_{3}\right)_{2}\right], 9.69$ and 10.15 (2 br s, $1 \mathrm{H}, \mathrm{NH}, \mathrm{D}_{2} \mathrm{O}$ exch.), 12.50 (br s, $1 \mathrm{H}, \mathrm{NH}, \mathrm{D}_{2} \mathrm{O}$ exch.) ppm; ${ }^{13} \mathrm{C}-\mathrm{NMR}\left(100 \mathrm{MHz}\right.$, DMSO- $\left.d_{6}\right) \delta 8.9,27.6,27.8,35.9,80.8,116.2,128.2,153.8$, 160.2, 162.9 ppm; IR (Nujol, $v, \mathrm{~cm}^{-1}$ ): 3188, 3115, 1741, 1645, 1607; MS m/z (ESI): 301.15 (M + H) ${ }^{+}$; calcd. for $\mathrm{C}_{12} \mathrm{H}_{20} \mathrm{~N}_{4} \mathrm{O}_{3} \mathrm{~S}$ (300.38): $\mathrm{C}, 47.98 ; \mathrm{H}, 6.71 ; \mathrm{N}, 18.65$; found: $\mathrm{C}, 48.11 ; \mathrm{H}, 6.63 ; \mathrm{N}, 18.57$. The partition of some signals here, as well as in the following cases, is due to the N1-amide rotameric effect [46].

\subsection{Typical MCR Procedure for the Synthesis of N-Substituted 1-Amino-1H-Imidazole-2(3H)-Thione Derivatives $5 \boldsymbol{a}-\boldsymbol{k}$}

To a round flask equipped with a magnetic stirring bar containing ice-cooled solution of $\mathrm{NaN}_{3}$ (1.0 mmol, $65.01 \mathrm{mg}$ ) dissolved in $0.5 \mathrm{~mL}$ of $\mathrm{H}_{2} \mathrm{O}$, the corresponding $\alpha$-halohydrazone 1a-k $(1.0 \mathrm{mmol})$ dissolved in THF $(4.5 \mathrm{~mL})$ was added. The mixture was stirred at room temperature until the disappearance of $\mathbf{1}$ (monitored by TLC). Upon completion, $\mathrm{Na}_{2} \mathrm{SO}_{4}(0.5 \mathrm{~g})$, a solution of $\mathrm{PPh}_{3}(1.1 \mathrm{mmol}$, $288.5 \mathrm{mg})$ in THF $(1.0 \mathrm{~mL})$ and $\mathrm{CS}_{2}(1.0 \mathrm{~mL})$ were added in sequence, and the mixture was refluxed for the appropriate reaction time (3.0-20.0 h). The formation of the final products $5 \mathbf{a}-\mathbf{k}$ was revealed by the complete disappearance of the spot corresponding to the $\alpha$-azidohydrazone $\mathbf{2} \mathbf{a}-\mathbf{k}$ as well as the detection of the byproduct $\mathrm{Ph}_{3} \mathrm{P}=\mathrm{S}$. The $\mathrm{Na}_{2} \mathrm{SO}_{4}$ was filtered in vacuo and washed with THF $(10.0 \mathrm{~mL})$. The filtrate was concentrated under reduced pressure and the residue was purified by crystallization and/or by chromatography eluting with cyclohexane:EtOAc or $\mathrm{CH}_{2} \mathrm{Cl}_{2}$ : $\mathrm{EtOAc}$ mixtures. The resulting products $5 \mathbf{a}-\mathbf{k}$ were isolated by crystallization from the specific solvents (see below). According to this procedure, $\mathbf{5 a}$ was obtained in $79 \%(237.3 \mathrm{mg})$.

N,N,5-trimethyl-1-(3-phenylureido)-2-thioxo-2,3-dihydro-1H-imidazole-4-carboxamide (5b): Yield 53\% (169.3 mg), pink powder from $\mathrm{CH}_{2} \mathrm{Cl}_{2} / \mathrm{Et}_{2} \mathrm{O} ; \mathrm{Mp} 247-248{ }^{\circ} \mathrm{C}$ (dec.); ${ }^{1} \mathrm{H}-\mathrm{NMR}\left(400 \mathrm{MHz}\right.$, DMSO-d $\left.\mathrm{d}_{6}\right) \delta 2.06(\mathrm{~s}, 3 \mathrm{H}$, $\left.\mathrm{CH}_{3}\right), 2.97\left[\mathrm{~s}, 6 \mathrm{H}, \mathrm{N}\left(\mathrm{CH}_{3}\right)_{2}\right], 7.01(\mathrm{t}, J=8.0 \mathrm{~Hz}, 1 \mathrm{H}, \mathrm{Ar}), 7.29(\mathrm{t}, J=8.0 \mathrm{~Hz}, 2 \mathrm{H}, \mathrm{Ar}), 7.46(\mathrm{~d}, J=8.0 \mathrm{~Hz}$, $2 \mathrm{H}, \mathrm{Ar}$ ), 9.00 (s, 1H, NH, $\mathrm{D}_{2} \mathrm{O}$ exch.), 9.33 (br s, $1 \mathrm{H}, \mathrm{NH}, \mathrm{D}_{2} \mathrm{O}$ exch.), 12.56 (s, $1 \mathrm{H}, \mathrm{NH}, \mathrm{D}_{2} \mathrm{O}$ exch.) ppm; ${ }^{13} \mathrm{C}-\mathrm{NMR}\left(100 \mathrm{MHz}, \mathrm{DMSO}-\mathrm{d}_{6}\right) \delta 9.3,36.8,116.1,118.3,122.3,128.7,129.0,139.1,153.6,160.3$, 162.6 ppm; IR (Nujol, v, cm ${ }^{-1}$ ): 3323, 3248, 3195, 3136, 1713, 1638, 1605; MS m/z (ESI): $320.40(\mathrm{M}+\mathrm{H})^{+}$; calcd. for; $\mathrm{C}_{14} \mathrm{H}_{17} \mathrm{~N}_{5} \mathrm{O}_{2} \mathrm{~S}$ (319.38): C, 52.65; $\mathrm{H}, 5.37 ; \mathrm{N}, 21.93$; calcd. for; $\mathrm{C}_{14} \mathrm{H}_{17} \mathrm{~N}_{5} \mathrm{O}_{2} \mathrm{~S}$ (319.38): $\mathrm{C}$, $52.65 ; \mathrm{H}, 5.37 ; \mathrm{N}, 21.93$; found: $\mathrm{C}, 52.79 ; \mathrm{H}, 5.44 ; \mathrm{N}, 21.84$.

tert-Butyl (4-(diethylcarbamoyl)-5-methyl-2-thioxo-2,3-dihydro-1H-imidazol-1-yl)carbamate (5c): Yield 72\% $(236.3 \mathrm{mg})$, white powder from EtOAc/THF/light petroleum ether; Mp $168-169{ }^{\circ} \mathrm{C}$ (dec.); ${ }^{1} \mathrm{H}-\mathrm{NMR}$ $\left(400 \mathrm{MHz}, \mathrm{DMSO}-\mathrm{d}_{6}\right) \delta 1.06-1.10\left(\mathrm{~m}, 6 \mathrm{H}, 2 \mathrm{xNCH}_{2} \mathrm{CH}_{3}\right), 1.32$ and $1.45\left(2 \mathrm{~s}, 9 \mathrm{H}, \mathrm{OBu}^{\mathrm{t}}\right), 1.94$ and $1.97(2 \mathrm{~s}$, $\left.3 \mathrm{H}, \mathrm{CH}_{3}\right), 3.26-3.37\left(\mathrm{~m}, 4 \mathrm{H}, 2 \times \mathrm{NCH}_{2} \mathrm{CH}_{3}\right), 9.68$ and 10.07 (2 br s, $1 \mathrm{H}, \mathrm{NH}, \mathrm{D}_{2} \mathrm{O}$ exch.), 12.49 (br s, $1 \mathrm{H}$, $\mathrm{NH}, \mathrm{D}_{2} \mathrm{O}$ exch.) ppm; ${ }^{13} \mathrm{C}-\mathrm{NMR}\left(100 \mathrm{MHz}, \mathrm{DMSO}_{6}\right.$ ) $\delta$ 8.6, 13.4, 27.5, 27.8, 34.8, 80.6, 117.0, 126.6, 153.8, 159.8, 162.7 ppm; IR (Nujol, $v, \mathrm{~cm}^{-1}$ ): 3169, 3120, 1748, 1642, 1634; MS m/z (ESI): 329.23 (M + $\mathrm{H})^{+}$; calcd. for $\mathrm{C}_{14} \mathrm{H}_{24} \mathrm{~N}_{4} \mathrm{O}_{3} \mathrm{~S}$ (328.16): C, 51.20; H, 7.37; N, 17.06; found: C, 51.09; H, 7.42; N, 16.95 . 
tert-Butyl (5-methyl-2-thioxo-2,3-dihydro-1H-imidazol-1-yl)carbamate (5d): Yield 69\% (158.1 mg), white powder from EtOAc/THF/light petroleum ether; Mp 168-169 ${ }^{\circ} \mathrm{C}$ (dec.); ${ }^{1} \mathrm{H}-\mathrm{NMR}(400 \mathrm{MHz}$, DMSO-d 6 ) $\delta 1.32$ and $1.45\left(2 \mathrm{~s}, 9 \mathrm{H}, \mathrm{OBu}^{\mathrm{t}}\right), 1.93\left(\mathrm{~s}, 3 \mathrm{H}, \mathrm{CH}_{3}\right), 6.60(\mathrm{~s}, 1 \mathrm{H}, \mathrm{CH}), 9.51$ and $9.94\left(2 \mathrm{br} \mathrm{s}, 1 \mathrm{H}, \mathrm{NH}, \mathrm{D}_{2} \mathrm{O}\right.$ exch.), 11.97 (br s, $1 \mathrm{H}, \mathrm{NH}, \mathrm{D}_{2} \mathrm{O}$ exch.) ppm; ${ }^{13} \mathrm{C}-\mathrm{NMR}$ (100 MHz, DMSO-d 6 ) 8 8.9, 27.9, 80.5, 108.9, 126.9, 153.9, 162.4 ppm; IR (Nujol, $v, \mathrm{~cm}^{-1}$ ): 3271, 3144, 3098, 1744, 1732, 1640; MS m/z (ESI): 229.96 (M $+\mathrm{H})^{+}$; calcd. for $\mathrm{C}_{9} \mathrm{H}_{15} \mathrm{~N}_{3} \mathrm{O}_{2} \mathrm{~S}(229.09): \mathrm{C}, 47.14 ; \mathrm{H}, 6.59 ; \mathrm{N}, 18.33$; found: $\mathrm{C}, 47.01 ; \mathrm{H}, 6.65 ; \mathrm{N}, 18.41$.

tert-Butyl (4-carbamoyl-5-methyl-2-thioxo-2,3-dihydro-1H-imidazol-1-yl)carbamate (5e): Yield 58\% (157.8 mg), white powder from $\mathrm{CH}_{2} \mathrm{Cl}_{2 /}$ light petroleum ether; $\mathrm{Mp} 270{ }^{\circ} \mathrm{C}$ (dec.); ${ }^{1} \mathrm{H}-\mathrm{NMR}$ (400 MHz, DMSO-d 6 ) $\delta$ 1.32 and $1.45\left(2 \mathrm{~s}, 9 \mathrm{H}, \mathrm{OBu}^{\mathrm{t}}\right), 2.23$ and $2.26\left(2 \mathrm{~s}, 3 \mathrm{H}, \mathrm{CH}_{3}\right), 7.23$ and $7.53\left(2 \mathrm{br} \mathrm{s}, 2 \mathrm{H}, \mathrm{NH}_{2}, \mathrm{D}_{2} \mathrm{O}\right.$ exch.), 9.71 and $10.17\left(2 \mathrm{~s}, 1 \mathrm{H}, \mathrm{NH}, \mathrm{D}_{2} \mathrm{O}\right.$ exch.), $12.42\left(\mathrm{~s}, 1 \mathrm{H}, \mathrm{NH}, \mathrm{D}_{2} \mathrm{O}\right.$ exch.) ppm; ${ }^{13} \mathrm{C}$ NMR (100 MHz, DMSO-d 6 )

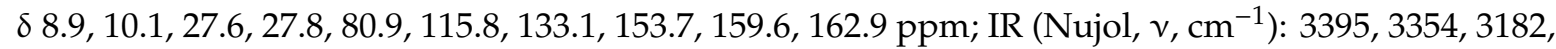
3137, 1754, 1717, 1676, 1594; MS m/z (ESI): $273.04(\mathrm{M}+\mathrm{H})^{+}$; calcd. for $\mathrm{C}_{10} \mathrm{H}_{16} \mathrm{~N}_{4} \mathrm{O}_{3} \mathrm{~S}$ (272.09): C, 44.10; H, 5.92; N, 20.57; found: C, 44.23; H, 5.96; N, 20.45.

tert-Butyl (5-methyl-4-(phenylcarbamoyl)-2-thioxo-2,3-dihydro-1H-imidazol-1-yl)carbamate (5f): Yield 67\% (233.2 mg), white powder from EtOAc; Mp 170-171 ${ }^{\circ} \mathrm{C}$ (dec.); ${ }^{1} \mathrm{H}-\mathrm{NMR}(400 \mathrm{MHz}$, DMSO-d 6 ) $\delta 1.34$ and $1.46\left(2 \mathrm{~s}, 9 \mathrm{H}, \mathrm{OBu}^{\mathrm{t}}\right), 2.28\left(\mathrm{~s}, 3 \mathrm{H}, \mathrm{CH}_{3}\right), 7.11(\mathrm{t}, J=8.0 \mathrm{~Hz}, 1 \mathrm{H}, \mathrm{Ar}), 7.35(\mathrm{t}, J=8.0 \mathrm{~Hz}, 2 \mathrm{H}, \mathrm{Ar}), 7.65(\mathrm{~d}$, $J=8.0 \mathrm{~Hz}, 2 \mathrm{H}, \mathrm{Ar}), 9.68$ (s, 1H, NH, $\mathrm{D}_{2} \mathrm{O}$ exch.), 10.28 (s, 1H, NH, $\mathrm{D}_{2} \mathrm{O}$ exch.), 12.69 (s, $1 \mathrm{H}, \mathrm{NH}, \mathrm{D}_{2} \mathrm{O}$ exch.) ppm; ${ }^{13} \mathrm{C}-\mathrm{NMR}\left(100 \mathrm{MHz}, \mathrm{DMSO}_{6}\right) \delta$ 9.2, 27.8, 81.0, 116.0, 119.7, 123.8, 128.8, 133.9, 138.4, 153.7, 156.3, 163.2 ppm; IR (Nujol, v, $\mathrm{cm}^{-1}$ ): 3375, 3243, 3066, 1752, 1659, 1630, 1598, 1545; MS m/z (ESI): $349.22(\mathrm{M}+\mathrm{H})^{+}$; calcd. for $\mathrm{C}_{16} \mathrm{H}_{20} \mathrm{~N}_{4} \mathrm{O}_{3} \mathrm{~S}$ (348.13): $\mathrm{C}, 55.16 ; \mathrm{H}, 5.79 ; \mathrm{N}, 16.08$; found: $\mathrm{C}, 55.01 ; \mathrm{H}, 5.72$; $\mathrm{N}, 16.16$.

1-(5-Methyl-2-thioxo-2,3-dihydro-1H-imidazol-1-yl)-3-phenylurea (5g): Yield 82\% (203.4 mg), white powder from THF/EtOAc; Mp 245-248 ${ }^{\circ} \mathrm{C}$ (dec.); ${ }^{1} \mathrm{H}-\mathrm{NMR}\left(400 \mathrm{MHz}, \mathrm{DMSO}-\mathrm{d}_{6}\right) \delta 2.01$ (s, 3H, $\left.\mathrm{CH}_{3}\right), 6.64$ (s, $1 \mathrm{H}, \mathrm{CH}), 6.99(\mathrm{t}, J=8.0 \mathrm{~Hz}, 1 \mathrm{H}, \mathrm{Ar}), 7.28(\mathrm{t}, J=8.0 \mathrm{~Hz}, 2 \mathrm{H}, \mathrm{Ar}), 7.46(\mathrm{~d}, J=8.0 \mathrm{~Hz}, 2 \mathrm{H}, \mathrm{Ar}), 8.91(\mathrm{~s}, 1 \mathrm{H}$, $\mathrm{NH}, \mathrm{D}_{2} \mathrm{O}$ exch.), 9.25 (s, 1H, NH, $\mathrm{D}_{2} \mathrm{O}$ exch.), 12.05 (s, $1 \mathrm{H}, \mathrm{NH}, \mathrm{D}_{2} \mathrm{O}$ exch.) ppm; ${ }^{13} \mathrm{C}-\mathrm{NMR}(100 \mathrm{MHz}$, DMSO-d $_{6}$ ) $\delta 9.1,108.8,118.3,122.3,127.6,128.8,139,1,153.8,161.9$ ppm; IR (Nujol, $v, \mathrm{~cm}^{-1}$ ): 3305, 3154, 3119, 3097, 1714, 1681, 1637, 1602; MS m/z (ESI): $249.07(\mathrm{M}+\mathrm{H})^{+}$; calcd. for $\mathrm{C}_{11} \mathrm{H}_{12} \mathrm{~N}_{4} \mathrm{OS}$ (248.07): C, 53.21; H, 4.87; N, 22.56; found: C, 53.08; H, 4.94; N, 22.65.

$\mathrm{N}$-(5-methyl-2-thioxoimidazolidin-1-yl)benzamide (5h): Yield 59\% (137.6 mg) white powder from $\mathrm{MeOH}$; Mp 240-242 ${ }^{\circ} \mathrm{C}$ (dec.); ${ }^{1} \mathrm{H}-\mathrm{NMR}\left(400 \mathrm{MHz}, \mathrm{DMSO}_{-} \mathrm{d}_{6}\right) \delta 1.97\left(\mathrm{~s}, 3 \mathrm{H}, \mathrm{CH}_{3}\right), 6.72(\mathrm{~s}, 1 \mathrm{H}, \mathrm{CH}), 7.56(\mathrm{t}$, $J=8.0 \mathrm{~Hz}, 2 \mathrm{H}, \mathrm{Ar}), 7.65(\mathrm{t}, J=8.0 \mathrm{~Hz}, 1 \mathrm{H}, \mathrm{Ar}), 7.99(\mathrm{~d}, J=8.0 \mathrm{~Hz}, 2 \mathrm{H}, \mathrm{Ar}), 11.44\left(\mathrm{~s}, 1 \mathrm{H}, \mathrm{NH}, \mathrm{D}_{2} \mathrm{O}\right.$ exch.), 12.15 (s, $1 \mathrm{H}, \mathrm{NH}, \mathrm{D}_{2} \mathrm{O}$ exch.) ppm; ${ }^{13} \mathrm{C}-\mathrm{NMR}\left(100 \mathrm{MHz}, \mathrm{DMSO}-\mathrm{d}_{6}\right) \delta 8.9,109.3,127.0,127.7,128.6$, 131.5, 132.5, 162.0, 165.4 ppm; IR (Nujol, $v, \mathrm{~cm}^{-1}$ ): 3168, 3106, 1666, 1631; MS m/z (ESI): 234.04 (M + $\mathrm{H})^{+}$; calcd. for $\mathrm{C}_{11} \mathrm{H}_{11} \mathrm{~N}_{3} \mathrm{OS}$ (233.29): C, 56.63; $\mathrm{H}, 4.75 ; \mathrm{N}, 18.01$; found: $\mathrm{C}, 56.76 ; \mathrm{H}, 4.82 ; \mathrm{N}, 17.89$.

1-(4,5-Dimethyl-2.thioxo-2,3-dihydro-1H-imidazol-1-yl)-3-phenylurea (5i): Yield 85\% (223.0 mg), white powder from THF/Et $2 \mathrm{O} ; \mathrm{Mp} 245-250{ }^{\circ} \mathrm{C}$ (dec.); ${ }^{1} \mathrm{H}-\mathrm{NMR}\left(400 \mathrm{MHz}, \mathrm{DMSO}-\mathrm{d}_{6}\right) \delta 1.94\left(\mathrm{~s}, 3 \mathrm{H}, \mathrm{CH}_{3}\right)$, $1.99\left(\mathrm{~s}, 3 \mathrm{H}, \mathrm{CH}_{3}\right), 6.99(\mathrm{t}, J=8.0 \mathrm{~Hz}, 2 \mathrm{H}, \mathrm{Ar}), 7.28(\mathrm{t}, J=8.0 \mathrm{~Hz}, 1 \mathrm{H}, \mathrm{Ar}), 7.46(\mathrm{~d}, J=8.0 \mathrm{~Hz}, 2 \mathrm{H}, \mathrm{Ar}), 8.89$ (s, $1 \mathrm{H}, \mathrm{NH}, \mathrm{D}_{2} \mathrm{O}$ exch.), 9.19 (s, $1 \mathrm{H}, \mathrm{NH}, \mathrm{D}_{2} \mathrm{O}$ exch.), 12.00 (s, $1 \mathrm{H}, \mathrm{NH}, \mathrm{D}_{2} \mathrm{O}$ exch.) ppm; ${ }^{13} \mathrm{C}-\mathrm{NMR}$ $\left(100 \mathrm{MHz}, \mathrm{DMSO}-\mathrm{d}_{6}\right) \delta 7.8,8.9,116.6,118.3,122.2,122.5,128.7,139.1,153.9,160.7$ ppm; IR (Nujol, $v$, $\mathrm{cm}^{-1}$ ): 3271, 3172, 3095, 1719, 1691, 1665, 1603; MS m/z (ESI): $263.11(\mathrm{M}+\mathrm{H})^{+}$; calcd. for $\mathrm{C}_{12} \mathrm{H}_{14} \mathrm{~N}_{4} \mathrm{OS}$ (262.33): C, 54.94; H, 5.38; N, 21.36; found: C, 54.87; H, 5.46; N, 21.23.

4,5-Dimethyl-1-[(4-nitrophenyl)amino]-1H-imidazole-2(3H)-thione (5j): Yield 84\% (222.0 mg), beige powder from THF/EtOAc/Et 2 O; Mp 279-282 ${ }^{\circ} \mathrm{C}$ (dec.); ${ }^{1} \mathrm{H}-\mathrm{NMR}\left(400 \mathrm{MHz}, \mathrm{DMSO}-\mathrm{d}_{6}\right) \delta 1.90\left(\mathrm{~s}, 3 \mathrm{H}, \mathrm{CH}_{3}\right), 2.03$ $\left(\mathrm{s}, 3 \mathrm{H}, \mathrm{CH}_{3}\right), 6.59$ (d, J = 8.0 Hz, 2H, Ar), $8.10(\mathrm{~d}, J=8.0 \mathrm{~Hz}, 2 \mathrm{H}, \mathrm{Ar}), 10.09$ (s, $1 \mathrm{H}, \mathrm{NH}, \mathrm{D}_{2} \mathrm{O}$ exch.), 12.19 (s, 1H, NH, $\mathrm{D}_{2} \mathrm{O}$ exch.) ppm; ${ }^{13} \mathrm{C}-\mathrm{NMR}\left(100 \mathrm{MHz}, \mathrm{DMSO}_{\mathrm{d}}\right.$ ) $\delta$ 7.6, 9.0, 111.3, 117.8, 121.7, 125.8, 139.3, 153.0, 160.8 ppm; IR (Nujol, $v, \mathrm{~cm}^{-1}$ ): 3199, 3094, 1673, 1594; HRMS m/z calcd. for $[\mathrm{M}+\mathrm{H}]^{+}$ $\mathrm{C}_{11} \mathrm{H}_{13} \mathrm{~N}_{4} \mathrm{O}_{2} \mathrm{~S}$ 265.0759; found 265.0774. 
tert-Butyl (5-phenyl-2-thioxo-2,3-dihydro-1H-imidazol-1-yl)carbamate (5k): Yield 66\% (192.3 mg), light yellow powder from THF/EtOAc/light petroleum ether; Mp 172-174 ${ }^{\circ} \mathrm{C}$ (dec.); ${ }^{1} \mathrm{H}-\mathrm{NMR}(400 \mathrm{MHz}$, DMSO- $\left._{6}\right) \delta 1.17$ and $1.39\left(2 \mathrm{~s}, 9 \mathrm{H}, \mathrm{OBu}^{\mathrm{t}}\right), 7.18(\mathrm{~s}, 1 \mathrm{H}, \mathrm{CH}), 7.35-7.49(\mathrm{~m}, 5 \mathrm{H}, \mathrm{Ar}), 9.79$ and $10.12(2 \mathrm{~s}, 1 \mathrm{H}$, $\mathrm{NH}, \mathrm{D}_{2} \mathrm{O}$ exch.), 12.51 (br s, $1 \mathrm{H}, \mathrm{NH}, \mathrm{D}_{2} \mathrm{O}$ exch.) ppm; ${ }^{13} \mathrm{C}-\mathrm{NMR}\left(100 \mathrm{MHz}, \mathrm{DMSO}-\mathrm{d}_{6}\right) \delta 27.5,27.9$, 80.2, 80.5, 110.7, 110.8, 126.9, 127.0, 127.7, 127.9, 128.1, 128.5, 130.6, 130.8, 153.2, 153.9, 162.0, 164.2 ppm; IR (Nujol, $v, \mathrm{~cm}^{-1}$ ): 3275, 3120, 3093, 1726, 1618, 1600; MS m/z (ESI): $292.18(\mathrm{M}+\mathrm{H})^{+}$; calcd. for $\mathrm{C}_{14} \mathrm{H}_{17} \mathrm{~N}_{3} \mathrm{O}_{2} \mathrm{~S}$ (291.37); C, 57.71; H, 5.88; N, 14.42; found: C, 57.83; H, 5.82; N, 14.37.

\subsection{General Procedure for the Synthesis of $\alpha$-(Imidazol-2-Ylthio) Carbonyl Compounds $7 \boldsymbol{a}-\boldsymbol{c}$.}

To a suspension of the $N$-Boc-protected 1 -amino- $1 H$-imidazole-2(3H)-thione derivatives $\mathbf{5} \mathbf{c}, \mathbf{d}, \mathbf{f}$ $(1.0 \mathrm{mmol})$ and $\mathrm{K}_{2} \mathrm{CO}_{3}(1.0 \mathrm{mmol}, 138 \mathrm{mg})$ in $10.0 \mathrm{~mL}$ of acetone, the corresponding $\alpha$-halocarbonyl derivative $6 \mathbf{a}-\mathbf{c}(1.0 \mathrm{mmol})$ was added. The reaction mixture was kept under magnetic stirring at room temperature. Upon completion (monitored by TLC) the solvent was removed, and the crude reaction mixture was quenched to neutrality with a solution of $\mathrm{HCl} 1 \mathrm{~N}$ and extracted with EtOAc $(30.0 \mathrm{~mL})$. The organic layer was washed with brine and dried over anhydrous $\mathrm{Na}_{2} \mathrm{SO}_{4}$. The solvent was removed in vacuum and the crude extract was purified by crystallization or by column chromatography eluting with cyclohexane:ethyl acetate mixtures to furnish $7 \mathbf{a}-\mathbf{c}$ derivatives in good yields ( $84 \%-93 \%)$.

tert-Butyl (4-(diethylcarbamoyl)-5-methyl-2-((2-oxo-2-phenylethyl)thio)-1H-imidazol-1-yl)carbamate (7a): Yield 84\% (375.1 mg); white solid from $\mathrm{Et}_{2} \mathrm{O}$; $\mathrm{Mp} 123-126{ }^{\circ} \mathrm{C} ;{ }^{1} \mathrm{H}-\mathrm{NMR}\left(400 \mathrm{MHz}, \mathrm{CDCl}_{3}\right) \delta 1.14-1.23$ $\left(\mathrm{m}, 6 \mathrm{H}, 2 \mathrm{NNCH}_{2} \mathrm{CH}_{3}\right), 1.48\left(\mathrm{~s}, 9 \mathrm{H}, \mathrm{OBu}^{\mathrm{t}}\right), 1.98\left(\mathrm{~s}, 3 \mathrm{H}, \mathrm{CH}_{3}\right), 3.37-3.64\left(\mathrm{~m}, 4 \mathrm{H}, 2 \mathrm{xNCH}_{2} \mathrm{CH}_{3}\right), 4.50(\mathrm{br} \mathrm{s}$, $\left.1 \mathrm{H}, \mathrm{SCH}_{\mathrm{a}} \mathrm{H}_{\mathrm{b}}\right), 4.69\left(\mathrm{br} \mathrm{s}, 1 \mathrm{H}, \mathrm{SCH}_{\mathrm{a}} \mathrm{H}_{\mathrm{b}}\right), 7.46(\mathrm{t}, J=8.0 \mathrm{~Hz}, 2 \mathrm{H}, \mathrm{Ar}), 7.58(\mathrm{t}, J=8.0 \mathrm{~Hz}, 1 \mathrm{H}, \mathrm{Ar}), 7.97(\mathrm{~d}$, $J=8.0 \mathrm{~Hz}, 2 \mathrm{H}, \mathrm{Ar}), 9.45$ (br s, $1 \mathrm{H}, \mathrm{NH}, \mathrm{D}_{2} \mathrm{O}$ exch.) ppm; ${ }^{13} \mathrm{C}-\mathrm{NMR}\left(100 \mathrm{MHz}, \mathrm{CDCl}_{3}\right) \delta 8.7,12.9,14.5$, 28.0, 40.6, 41.6, 43.4, 82.5, 128.4, 128.5, 128.7, 130.9, 133.7, 135.3, 135.4, 139.9, 153.8, 164.5, 193.7 ppm; IR (Nujol, $v, \mathrm{~cm}^{-1}$ ): 3114, 3059, 1741, 1726, 1700, 1681, 1597, 1584; MS m/z (ESI): 447.35 (M + H) ${ }^{+}$; calcd. for $\mathrm{C}_{22} \mathrm{H}_{30} \mathrm{~N}_{4} \mathrm{O}_{4} \mathrm{~S}$ (446.56): C, 59.17; $\mathrm{H}, 6.77 ; \mathrm{N}, 12.55$; found: $\mathrm{C}, 59.02 ; \mathrm{H}, 6.84 ; \mathrm{N}, 11.67$.

tert-Butyl (5-methyl-2-((2-oxopropyl)thio)-1H-imidazol-1-yl)carbamate (7b): Yield 93\% (265.4 mg); ocher solid from EtOAc/cyclohexane; Mp 103-104 ${ }^{\circ} \mathrm{C} ;{ }^{1} \mathrm{H}-\mathrm{NMR}\left(400 \mathrm{MHz}, \mathrm{CDCl}_{3}\right) \delta 1.50\left(\mathrm{~s}, 9 \mathrm{H}, \mathrm{OBu}^{\mathrm{t}}\right), 2.14$ $\left(\mathrm{s}, 3 \mathrm{H}, \mathrm{CH}_{3}\right), 2.24\left(\mathrm{~s}, 3 \mathrm{H}, \mathrm{COCH}_{3}\right), 3.81\left(\mathrm{br} \mathrm{s}, 1 \mathrm{H}, \mathrm{SCH}_{\mathrm{a}} \mathrm{H}_{\mathrm{b}}\right), 3.94\left(\mathrm{br} \mathrm{s}, 1 \mathrm{H}, \mathrm{SCH}_{\mathrm{a}} \mathrm{H}_{\mathrm{b}}\right), 6.77(\mathrm{~s}, 1 \mathrm{H}, \mathrm{CH})$, 8.26 (br s, $1 \mathrm{H}, \mathrm{NH}, \mathrm{D}_{2} \mathrm{O}$ exch.) ppm; ${ }^{13} \mathrm{C}-\mathrm{NMR}\left(100 \mathrm{MHz}, \mathrm{CDCl}_{3}\right) \delta 8.9,28.0,28.8,44.8,82.8,125.1$, 131.5, 139.5, 154.1, 203.5 ppm; IR (Nujol, $v, \mathrm{~cm}^{-1}$ ): 3125, 1725, 1714; MS m/z (ESI): $286.17(\mathrm{M}+\mathrm{H})^{+}$; calcd. for $\mathrm{C}_{12} \mathrm{H}_{19} \mathrm{~N}_{3} \mathrm{O}_{3} \mathrm{~S}$ (285.36): C, 50.51; H, 6.71; N, 14.73; found: $\mathrm{C}, 50.40 ; \mathrm{H}, 6.78 ; \mathrm{N}, 14.86$.

Ethyl 2-((1-((tert-Butoxycarbonyl)amino)-5-methyl-4-(phenylcarbamoyl)-1H-imidazol-2-yl)thio)acetate (7c): Yield 92\% (399.7 mg), white solid from EtOAc/cyclohexane; Mp $134-136{ }^{\circ} \mathrm{C} ;{ }^{1} \mathrm{H}-\mathrm{NMR}(400 \mathrm{MHz}$, $\left.\mathrm{CDCl}_{3}\right) \delta 1.27\left(\mathrm{t}, J=8.0 \mathrm{~Hz}, 3 \mathrm{H}, \mathrm{OCH}_{2} \mathrm{CH}_{3}\right), 1.51\left(\mathrm{~s}, 9 \mathrm{H}, \mathrm{OBu}^{\mathrm{t}}\right), 2.55\left(\mathrm{~s}, 3 \mathrm{H}, \mathrm{CH}_{3}\right), 3.66(\mathrm{~d}, J=16.0 \mathrm{~Hz}$, $\left.1 \mathrm{H}, \mathrm{SCH}_{\mathrm{a}} \mathrm{H}_{\mathrm{b}}\right), 3.90\left(\mathrm{~d}, J=16.0 \mathrm{~Hz}, 1 \mathrm{H}, \mathrm{SCH}_{\mathrm{a}} \mathrm{H}_{\mathrm{b}}\right), 4.17-4.24\left(\mathrm{~m}, 2 \mathrm{H}, \mathrm{OCH}_{2} \mathrm{CH}_{3}\right), 7.10(\mathrm{t}, J=8.0 \mathrm{~Hz}, 1 \mathrm{H}$, $\mathrm{Ar}), 7.34(\mathrm{t}, J=8.0 \mathrm{~Hz}, 2 \mathrm{H}, \mathrm{Ar}), 7.67(\mathrm{~d}, J=8.0 \mathrm{~Hz}, 2 \mathrm{H}, \mathrm{Ar}), 8.18\left(\mathrm{br} \mathrm{s}, 1 \mathrm{H}, \mathrm{NH}, \mathrm{D}_{2} \mathrm{O}\right.$ exch.), $8.96(\mathrm{~s}, 1 \mathrm{H}$, $\mathrm{NH}, \mathrm{D}_{2} \mathrm{O}$ exch.) ppm; ${ }^{13} \mathrm{C}-\mathrm{NMR}\left(100 \mathrm{MHz}, \mathrm{CDCl}_{3}\right): \delta$ 9.4, 14.0, 28.0, 36.7, 62.7, 83.4, 119.5, 123.8, 128.9, 129.9, 137.3, 138.0, 139.0, 153.6, 160.6, 169.9 ppm; IR (Nujol, v, $\mathrm{cm}^{-1}$ ): 3315, 3182, 1733, 1647, 1601; MS $m / z$ (ESI): $435.20(\mathrm{M}+\mathrm{H})^{+}$; calcd. for $\mathrm{C}_{20} \mathrm{H}_{26} \mathrm{~N}_{4} \mathrm{O}_{5} \mathrm{~S}$ (434.51): C, 55.28; $\mathrm{H}, 6.03 ; \mathrm{N}, 12.89$; found: $\mathrm{C}, 55.39$; $\mathrm{H}, 5.97 ; \mathrm{N}, 12.81$.

\subsection{General Procedure for the Synthesis of N-Bridgeheaded Heterobicyclic Derivatives $\mathbf{8 a}-\boldsymbol{c}$.}

Derivative $7 \mathbf{a}, \mathbf{b}(1.0 \mathrm{mmol})$ was solved in $5.0 \mathrm{~mL}$ of a solution of trifluoroacetic acid (TFA) and $\mathrm{CH}_{2} \mathrm{Cl}_{2}$ (1:1). The reaction mixture was left at room temperature until the disappearance of the starting $\mathbf{7 a}, \mathbf{b}$ (TLC check). Then, the solvent was removed under reduced pressure and the crude reaction mixture was quenched to neutrality with a saturated solution of $\mathrm{Na}_{2} \mathrm{CO}_{3}$ and extracted with EtOAc $(20.0 \mathrm{~mL} \times 3)$. The combined organic layers were washed with brine and dried over anhydrous $\mathrm{Na}_{2} \mathrm{SO}_{4}$. After the removal of the solvent, the crude extract was purified by crystallization or by column chromatography eluting with cyclohexane/ethyl acetate mixtures to furnish $\mathbf{8 a}, \mathbf{b}$ derivatives. 
For obtaining $8 \mathbf{c}$, the best condition found was to treat $7 \mathrm{c}(1.0 \mathrm{mmol})$ with Amberlyst $15 \mathrm{H}(500 \mathrm{mg})$ in refluxing dioxane $(15.0 \mathrm{~mL}$ ) for $12.0 \mathrm{~h}$. Upon completion (monitored by TLC) the resin was filtered off in vacuo and washed with THF $(20.0 \mathrm{~mL})$. The filtrate was evaporated under reduced pressure and the crude reaction mixture was purified by crystallization.

N,N-diethyl-6-methyl-3-phenyl-2H-imidazo[2,1-b][1,3,4]thiadiazine-7-carboxamide (8a): Yield 82\% (269.3 mg) white powder from EtOAc/cyclohexane; $\mathrm{Mp} 125-127^{\circ} \mathrm{C} ;{ }^{1} \mathrm{H}-\mathrm{NMR}\left(400 \mathrm{MHz}, \mathrm{CDCl}_{3}\right) \delta 1.22(\mathrm{t}, J=8.0 \mathrm{~Hz}$, $\left.6 \mathrm{H}, 2 \mathrm{xNCH}_{2} \mathrm{CH}_{3}\right), 2.56\left(\mathrm{~s}, 3 \mathrm{H}, \mathrm{CH}_{3}\right), 3.53$ (br s, $\left.2 \mathrm{H}, \mathrm{NCH}_{2} \mathrm{CH}_{3}\right), 3.74$ (br s, $\left.2 \mathrm{H}, \mathrm{NCH}_{2} \mathrm{CH}_{3}\right), 3.97(\mathrm{~s}, 2 \mathrm{H}$, $\left.\mathrm{SCH}_{2}\right), 7.50-7.52(\mathrm{~m}, 3 \mathrm{H}, \mathrm{Ar}), 7.90(\mathrm{~d}, J=8.0 \mathrm{~Hz}, 2 \mathrm{H}, \mathrm{Ar}) \mathrm{ppm} ;{ }^{13} \mathrm{C}-\mathrm{NMR}\left(100 \mathrm{MHz}, \mathrm{CDCl}_{3}\right) \delta$ 9.7, 13.0, 14.5, 23.9, 40.3, 43.0, 127.0, 128.9, 129.7, 131.1, 131.3, 133.5, 134.2, 150.8, 164.1 ppm; IR (Nujol, $\left.v, \mathrm{~cm}^{-1}\right)$ : 1611, 1574, 1562, 1557; MS m/z (ESI): $329.28(\mathrm{M}+\mathrm{H})^{+}$; calcd. for $\mathrm{C}_{17} \mathrm{H}_{20} \mathrm{~N}_{4} \mathrm{OS}$ (328.43): $\mathrm{C}, 62.17 ; \mathrm{H}, 6.14$; $\mathrm{N}, 17.06$; found: $\mathrm{C}, 62.04 ; \mathrm{H}, 6.19 ; \mathrm{N}, 17.15$.

3,6-Dimethyl-2H-imidazo[2,1-b][1,3,4]thiadiazine (8b): Yield 65\% (108.7 mg); white needles from $\mathrm{CHCl}_{3} /$ cyclohexane; Mp 57-58 ${ }^{\circ} \mathrm{C} ;{ }^{1} \mathrm{H}-\mathrm{NMR}\left(400 \mathrm{MHz}, \mathrm{CDCl}_{3}\right) \delta 2.26\left(\mathrm{~s}, 3 \mathrm{H}, \mathrm{CH}_{3}\right), 2.32\left(\mathrm{~s}, 3 \mathrm{H}, \mathrm{CH}_{3}\right), 3.42(\mathrm{~s}$, $\left.2 \mathrm{H}, \mathrm{SCH}_{2}\right), 6.70(\mathrm{~s}, 1 \mathrm{H}, \mathrm{CH}), \mathrm{ppm} ;{ }^{13} \mathrm{C}-\mathrm{NMR}\left(100 \mathrm{MHz}, \mathrm{CDCl}_{3}\right) \delta 8.8,23.5,26.1,123.7,128.6,130.1$, 152.0 ppm; IR (Nujol, $v, \mathrm{~cm}^{-1}$ ): 1640. 1582; MS m/z (ESI): $168.06(\mathrm{M}+\mathrm{H})^{+}$; calcd. for $\mathrm{C}_{7} \mathrm{H}_{9} \mathrm{~N}_{3} \mathrm{~S}(167.23)$ : C, 50.27; H, 5.42; N, 25.13; found: C, 50.39; H, 5.39 N, 25.06 .

6-Methyl-3-oxo-N-phenyl-3,4-dihydro-2H-imidazo[2,1-b][1,3,4]thiadiazine-7-carboxamide (8c): Yield 74\% (213.3 mg), light yellow powder from EtOAc/light petroleum ether; $\mathrm{Mp} 229-232{ }^{\circ} \mathrm{C} ;{ }^{1} \mathrm{H}-\mathrm{NMR}(400 \mathrm{MHz}$, DMSO-d $\left._{6}\right) \delta 2.53\left(\mathrm{~s}, 3 \mathrm{H}, \mathrm{CH}_{3}\right), 3.81\left(\mathrm{~s}, 2 \mathrm{H}, \mathrm{SCH}_{2}\right), 7.04(\mathrm{t}, J=8.0 \mathrm{~Hz}, 1 \mathrm{H}, \mathrm{Ar}), 7.29(\mathrm{t}, J=8.0 \mathrm{~Hz}, 2 \mathrm{H}, \mathrm{Ar})$, 7.81 (d, J = $8.0 \mathrm{~Hz}, 2 \mathrm{H}, \mathrm{Ar}), 9.80$ (s, $1 \mathrm{H}, \mathrm{NH}, \mathrm{D}_{2} \mathrm{O}$ exch.), 12.27 (br s, $1 \mathrm{H}, \mathrm{NH}, \mathrm{D}_{2} \mathrm{O}$ exch.) ppm; ${ }^{13} \mathrm{C}-\mathrm{NMR}$ $\left(100 \mathrm{MHz}, \mathrm{DMSO}_{\mathrm{d}}\right.$ ): $\delta$ 9.2, 29.7, 119.8, 123.0, 128.3, 128.4, 130.6, 132.2, 138.8, 160.8, 164.6 ppm; IR (Nujol, $v, \mathrm{~cm}^{-1}$ ): 1679, 1666, 1595, 1582; MS m/z (ESI): $288.97(\mathrm{M}+\mathrm{H})^{+}$; calcd. for $\mathrm{C}_{13} \mathrm{H}_{12} \mathrm{~N}_{4} \mathrm{O}_{2} \mathrm{~S}$ (288.32): C, 54.15; H, 4.20; N, 19.43; found: C, 54.08; H, 4.27; N, 19.31.

\section{Conclusions}

In conclusion, combining sequential azidation, Staudinger, and aza-Wittig reactions with $\mathrm{CS}_{2}$ on $\alpha$-halohydrazones in a one-pot protocol, variously substituted 1 -amino- $1 H$-imidazole- $2(3 H)$-thiones are directly accessible in good yields and with complete control of regioselectivity. The method is particularly attractive and advantageous for its mild conditions, operational simplicity, and its efficiency as well as its robustness (wide substrate scope and tolerance of various functional groups) and reliability. The concurrent presence of reactive appendages on the obtained scaffolds ensures post-modifications toward $\mathrm{N}$-bridgeheaded heterobicyclic structures.

Supplementary Materials: The following are available online: procedure followed for obtaining II; copies of ${ }^{1} \mathrm{H}-\mathrm{NMR}$ and ${ }^{13} \mathrm{C}$-NMR spectra of II [22]; copies of ${ }^{1} \mathrm{H}-\mathrm{NMR}$ and ${ }^{13} \mathrm{C}-\mathrm{MNR}$ spectra of all newly synthesized compounds; copies of HMQC of compound $\mathbf{5 a}$ and $\mathbf{8 c}$.

Author Contributions: C.C. performed all synthetic work in laboratory; G.M. designed the experiments; G.F.; supervised the project, F.M. funding acquisition; L.D.C. validation; S.S.* conceived the synthetic route and wrote the paper.

Funding: The authors declare no competing financial interest.

Acknowledgments: The authors gratefully thank Anna Maria Gioacchini and Samuele Lillini who competently performed the mass spectra and the Department of Biomolecular Science of the University of Urbino for the economical support.

Conflicts of Interest: There are no conflicts to declare.

\section{References}

1. Uçucu, O.; Karaburun, N.G.; Işikdağ, I. Synthesis and analgesic activity of some 1-benzyl-2-substituted-4,5diphenyl-1H-imidazole derivatives. Il Farmaco 2001, 56, 285-290. [CrossRef] 
2. Rani, N.; Sharma, A.; Gupta, G.K.; Singh, R. Imidazoles as potential antifungal agents: A review. Mini-Rev. Med. Chem. 2013, 13, 1626-1655. [CrossRef] [PubMed]

3. Khalafi-Nezhad, A.; Rad, M.N.S.; Mohabatkar, H.; Asrari, Z.; Hemmateenejad, B. Design, synthesis, antibacterial and QSAR studies of benzimidazole and imidazole chloroaryloxyalkyl derivatives. Bioorg. Med. Chem. 2005, 13, 1931-1938. [CrossRef]

4. Kerru, N.; Bhaskaruni, S.V.H.S.; Gummidi, L.; Maddila, S.N.; Maddila, S.; Jonnalagadda, S.B. Recent advances in heterogeneous catalysts for the synthesis of imidazole derivatives. Synthetic Commun. 2019, 49, 2437-2459. [CrossRef]

5. Ali, I.; Lone, M.N.; Aboul-Enein, H.Y. Imidazoles as potential anticancer agents. MedChem Comm 2017, 8, $1742-1773$. [CrossRef]

6. Sharma, A.; Kumar, V.; Kharb, R.; Kumar, S.; Sharma, P.C.; Pathak, D.P. Imidazole derivatives as potential therapeutic agents. Curr. Pharm. Des. 2016, 22, 3265-3301. [CrossRef]

7. Bellina, F.; Cauteruccio, S.; Rossi, R. Synthesis and biological activity of vicinal diaryl-substituted $1 H$-imidazoles. Tetrahedron 2007, 63, 4571-4624. [CrossRef]

8. Savjani, J.K.; Gajjar, A.K. Pharmaceutical importance and synthetic strategies for imidazolidine-2-thione and imidazole-2-thione derivatives. Pak. J. Biol. Sci. 2011, 14, 1076-1089. [CrossRef]

9. Isaia, F.; Aragoni, M.C.; Arca, M.; Demartin, F.; Devillanova, F.A.; Floris, G.; Garau, A.; Hursthouse, M.B.; Lippolis, V.; Medda, R.; et al. Interaction of methimazole with I2: X-ray crystal structure of the charge transfer complex methimazole-I2. implications for the mechanism of action of methimazole-based antithyroid drugs. Med. Chem. 2008, 51, 4050-4053. [CrossRef]

10. Cesarini, S.; Spallarossa, A.; Ranise, A.; Schenone, S.; Rosano, C.; La Colla, P.; Sanna, G.; Busonera, B.; Loddo, R. N-acylated and $N, N^{\prime}$-diacylated imidazolidine-2-thione derivatives and $N, N^{\prime}$-diacylated tetrahydropyrimidine-2(1H)-thione analogues: Synthesis and antiproliferative activity. Eur. J. Med. Chem. 2009, 44, 1106-1118. [CrossRef]

11. Sheppeck, J.; Gilmore, J.L. Substituted 1.3-dihydro-imidazol-2-one and 1.3-dihydro-imidazol-2-thione derivatives as inhibitors of matrix metallo proteinases and/or TNF- $\alpha$ converting enzyme (TACE). U.S. Patent 20050075384, 7 April 2005.

12. Lagoja, I.M.; Pannecouque, C.; Van Aerschot, A.; Witvrouw, M.; Debyser, Z.; Balzarini, J.; Herdewijn, P.; De Clercq, E.J. $N$-Aminoimidazole derivatives inhibiting retroviral replication via a yet unidentified mode of action. Med. Chem. 2003, 46, 1546-1553. [CrossRef] [PubMed]

13. Schantl, J.G.; Prean, M. Addition products of hydrazine derivatives to azo-alkenes, part V: The reaction of $\alpha$-(1-phenylhydrazino)alkanone phyenylhydrazones with acids and acid derivatives. Monatsh. Chem. 1993, 124, 299-308. [CrossRef]

14. Schantl, J.G.; Kahlig, H.; Preans, M. 1-Arylamino-2,3-dihydro-1H-imidazole-2-thiones from the reaction of 1-[2-(2-arylhydrazono)alkyl]pyridinium iodides with potassium thiocyanate. Heterocycles 1994, 37, 1873-1878. [CrossRef]

15. Schantl, J.G.; Lagoia, I. Direct synthetic approach to $\mathrm{N}$-Substituted 1-amino-2,3-dihydro-1H-imidazole2-thiones. Heterocycles 1997, 45, 691-700. [CrossRef]

16. Schantl, J.; Nádenik, P. Tandem [3+2] cycloaddition reaction of azo-alkenes and thiocyanic acid: Extending the scope of the classical "criss-cross" cycloaddition reaction. Synlett 1998, 786-788. [CrossRef]

17. Yurttaş, L.; Ertas, M.; Gulsen, A.C.; Temel, H.E.; Demirayak, Ş. Novel benzothiazole based imidazole derivatives as new cytotoxic agents against glioma (C6) and liver (HepG2) cancer cell lines. Acta Pharm. Sci. 2017, 55, 39-47. [CrossRef]

18. Neochoritis, C.; Tsoleridis, C.A.; Stephanidou-Stephanatou, J. 1-Arylaminoimidazole-2-thiones as intermediates in the synthesis of imidazo[2,1-b][1,3,4]thiadiazines. Tetrahedron 2008, 64, 3527-3533. [CrossRef]

19. Grimmett, M.R. Imidazoles. In Science of Synthesis; Neier, D., Bellus, D., Eds.; G. Thieme Verlag: Stuttgart, Germany, 2002; Volume 12, pp. 325-328. [CrossRef]

20. Schantl, J.G. Azomethine imines. In Science of Synthesis; Padwa, A., Ed.; G. Thieme Verlag: Stuttgart, Germany, 2004; Volume 27, pp. 731-738. [CrossRef]

21. Schantl, J.G. Cyclic azomethine imines from diazenes (azo compounds). In Advances in Heterocyclic Chemistry; Katritzky, A.R., Ed.; Academic Press: Oxford, UK; Volume 99, pp. 185-207. [CrossRef]

22. Attanasi, O.A.; Favi, G.; Filippone, P.; Perrulli, F.R.; Santeusanio, S. Direct access to variously substituted 2-imino-4-thiazolines. Synlett 2010, 1859-1861. [CrossRef] 
23. Attanasi, O.A.; Bartoccini, S.; Favi, G.; Filippone, P.; Perrulli, F.R.; Santeusanio, S. Tandem aza-Wittig/ carbodiimide-mediated annulation applicable to 1,2-diaza-1,3-dienes for the one-pot synthesis of fully substituted 1,2-diaminoimidazoles. J. Org. Chem. 2012, 77, 9338-9343. [CrossRef]

24. Xie, H.; Liu, J.-C.; Wu, L.; Ding, M.-W. Unexpected synthesis of 2,4,5-trisubstituted oxazoles via a tandem aza-Wittig/Michael/isomerization reaction of vinyliminophosphorane. Tetrahedron 2012, 68, 7984-7990. [CrossRef]

25. Santhosh, L.; Durgamma, S.; Sureshbabu, V.V. Staudinger/aza-Wittig reaction to access $N^{\beta}$-protected amino alkyl isothiocyanates. Org. Biomol. Chem. 2018, 16, 4874-4880. [CrossRef] [PubMed]

26. Lopes, S.M.M.; Lemos, A.; Pinho e Melo, T.M.V.D. Reactivity of dipyrromethanes towards azoalkenes: Synthesis of functionalized dipyrromethanes, calix[4]pyrroles, and bilanes. Eur. J. Org. Chem. 2014, 7039-7048. [CrossRef]

27. Attanasi, O.A.; De Crescentini, L.; Favi, G.; Filippone, P.; Mantellini, F.; Perrulli, F.R.; Santeusanio, S. Cultivating the passion to build heterocycles from 1,2-diaza-1,3-dienes: The force of imagination. Eur. J. Org. Chem. 2009, 3109-3127. [CrossRef]

28. Attanasi, O.A.; De Crescentini, L.; Giorgi, R.; Perrone, A.; Santeusanio, S. Synthesis of 3-unsubstituted1-aminopyrroles. Heterocycles 1996, 43, 1447-1457. [CrossRef]

29. Attanasi, O.A.; Favi, G.; Mantellini, F.; Mantenuto, S.; Moscatelli, G.; Nicolini, S. Regioselective formation of 5-methylene-6-methoxy-1,4,5,6-tetrahydropyridazines from the [4+2]-cycloaddition reaction of in situ generated 1,2-diaza-1,3-dienes with methoxyallene. Synlett 2015, 193-196. [CrossRef]

30. Attanasi, O.A.; De Crescentini, L.; Favi, G.; Mantellini, F.; Mantenuto, S.; Nicolini, S. Interceptive [4+1] annulation of in situ generated 1,2-diaza-1,3-dienes with diazo esters: Direct access to substituted mono-, bi-, and tricyclic 4,5-dihydropyrazoles. J. Org. Chem. 2014, 79, 8331-8338. [CrossRef]

31. Attanasi, O.A.; Serra-Zanetti, F.; Zhiyuan, L. Easy one-pot conversion of 2-chlorohydrazone into 2-oxohydrazone derivatives via 2-azidohydrazone intermediates. Tetrahedron 1992, 48, 2785-2792. [CrossRef]

32. Herrera, R.P.; Marqués-Lopez, E. Multicomponent Reactions: Concepts and Applications for Design and Synthesis; John Wiley \& Sons: Hoboken, NJ, USA, 2015.

33. Dömling, A. Recent developments in isocyanide based multicomponent reactions in applied chemistry. Chem. Rev. 2006, 106, 17-89. [CrossRef]

34. Dömling, A.; Wang, W.; Wang, K. Chemistry and biology of multicomponent reactions. Chem. Rev. 2012, 112, 3083-3135. [CrossRef]

35. Preeti; Singh, K.N. Multicomponent reactions: A sustainable tool to 1,2- and 1,3-azoles. Org. Biomol. Chem. 2018, 16, 9084-9116. [CrossRef] [PubMed]

36. Yurttaş, L.; Duran, M.; Demirayak, S.; Gençer, H.K.; Tunali, Y. Synthesis and initial biological evaluation of substituted 1-phenylamino-2-thio-4,5-dimethyl-1H-imidazole derivatives. Bioorg. Med. Chem. Lett. 2013, 23, 6764-6768. [CrossRef]

37. Palacios, F.; Alonso, C.; Aparicio, D.; Rubiales, G.; de los Santos, J.M. The aza-Wittig reaction: An efficient tool for the construction of carbon-nitrogen double bonds. Tetrahedron 2007, 63, 523-575. [CrossRef]

38. Eguchi, S. Recent progress in the synthesis of heterocyclic natural products by the Staudinger/intramolecular aza-Wittig reaction. ARKIVOC 2005, (ii), 98-119. [CrossRef]

39. Fresneda, P.M.; Molina, P. Application of iminophosphorane-based methodologies for the synthesis of natural products. Synlett 2004, 1-17. [CrossRef]

40. Pavlova, A.S.; Ianova, O.A.; Chagarovskij, A.O.; Stebunov, N.S.; Orlov, N.V.; Shumsky, A.N.; Budynina, E.M.; RybaKov, V.B.; Trushkov, I.V. Domino Staudinger/aza-Wittig/Mannich reaction: An approach to diversity of di- and tetrahydropyrrole scaffolds. Chem. Eur. J. 2016, 22, 17967-17971. [CrossRef]

41. Xiong, J.; Wei, X.; Ding, M.-W. New Facile synthesis of 2-alkylthiopyrimidin-4(3H)-ones by tandem aza-Wittig reaction starting from the Baylis-Hillman adducts. Synlett 2017, 1075-1078. [CrossRef]

42. Agami, C.; Couty, F. The reactivity of the N-Boc protecting group: An underrated feature. Tetrahedron 2002, 58, 2701-2724. [CrossRef]

43. Greene, T.W.; Wuts, P.G.M. Protective Groups in Organic Synthesis, 3rd ed.; Wiley: New York, NY, USA, 1999. [CrossRef]

44. Ballini, R.; Petrini, M. Amberlyst 15, a superior, mild, and selective catalyst for carbonyl regeneration from nitrogeneous derivatives. J. Chem. Soc. Perkin Trans. 1 1988, 2563-2565. [CrossRef] 
45. Sasaki, T.; Ito, E.; Shimizu, I. Ring transformations of oxazoles and their benzo analogues. New synthetic route for $2 H$-imidazo[2,1-b][1,3,4]thiadiazine and N-heteroaryl-o-aminophenol. Heterocycles 1982, 19, 2119-2129. [CrossRef]

46. Qi, L.-W.; Mao, J.-H.; Zhang, J.; Tan, B. Organocatalytic asymmetric arylation of indoles enabled by azo groups. Nat. Chem. 2018, 10, 58-64. [CrossRef] [PubMed]

Sample Availability: Samples of the compounds 5a-k are available from the authors.

(C) 2019 by the authors. Licensee MDPI, Basel, Switzerland. This article is an open access article distributed under the terms and conditions of the Creative Commons Attribution (CC BY) license (http://creativecommons.org/licenses/by/4.0/). 